\title{
Siderophore-specific induction of iron uptake in Pseudomonas aeruginosa
}

\author{
Karl Gensberg, Kevin Hughes and Anthony W. Smith* \\ Microbiology Research Group, Pharmaceutical Sciences Institute, Aston University, Birmingham B4 7ET, UK
}

(Received 14 May 1992; revised 20 July 1992; accepted 3 August 1992)

\begin{abstract}
Pseudomonas aeruginosa has two siderophore-based high-affinity iron-uptake systems utilizing pyoverdin and pyochelin. Using strain IA1, a mutant deficient in production of both siderophores, we have shown that addition of purified siderophore to the growth medium induces expression of specific iron-regulated outer-membrane proteins and increases ${ }^{55} \mathrm{Fe}$-siderophore transport. Addition of pyoverdin from the parent strain PAO1 or from a clinical strain 0:12 induced expression of an 85 kDa IROMP and increased the rate of ${ }^{55} \mathrm{Fe}$-pyoverdin transport. Transport rates for ${ }^{55} \mathrm{Fe}-\mathrm{PAO1}$ pyoverdin increased from 1.27 to $3.57 \mathrm{pmol} \mathrm{Fe} \mathrm{min}^{-1}$ per $10^{9}$ cells. Addition of purified pyochelin induced expression of a $75 \mathrm{kDa}$ IROMP accompanied with increased ${ }^{55} \mathrm{Fe}$-pyochelin uptake without affecting ${ }^{55} \mathrm{Fe}$-pyoverdin transport. ${ }^{55} \mathrm{Fe}$-pyochelin transport increased from 0.3 to $10.6 \mathrm{pmol} \mathrm{min}{ }^{-1}$ per $10^{9}$ cells. Addition of pyoverdin from the parent strain or a chromatographically distinct pyoverdin caused increased reactivity with an anti-85 $\mathrm{kDa} \mathrm{mAb}$ in Western blotting, indicating that the same receptor is being induced. These results suggest that $\boldsymbol{P}$. aeruginosa can respond specifically to the presence of siderophore and moreover that not only can the pyoverdin receptor transport its cognate ferri-pyoverdin but also different ferripyoverdins, albeit at a reduced rate.
\end{abstract}

\section{Introduction}

Pseudomonas aeruginosa has two siderophore-mediated iron uptake systems based on pyochelin and pyoverdin. These systems will operate both in the organism's natural habitat, soil and water, where the solubility of iron at neutral $\mathrm{pH}$ is extremely low, and in the human host where the availability of free iron is too low to sustain bacterial growth due to the iron-binding glycoproteins transferrin and lactoferrin (Griffiths, 1991).

Pyochelin comprises a salicyl ring bonded to a thiazoline ring, which is itself bonded to a $N$-methylthiazolidine ring (Cox et al., 1981), and appears to be produced by all strains. Two iron-regulated outermembrane protein (IROMP) uptake systems have been identified for this siderophore. Firstly, a $14 \mathrm{kDa}$ ferripyochelin binding protein was detected (Sokol \& Woods, 1983) and mutants deficient in surface expression of this protein showed reduced ferri-pyochelin uptake (Sokol, 1987). Secondly, a $75 \mathrm{kDa}$ IROMP has been shown to be associated with ferri-pyochelin uptake (Heinrichs et al., 1991) using protease protection and labelled-iron transport studies. Mutants deficient in expression either of the

* Author for correspondence. Tel. (021) 3593611 ext. 4770; fax (021) 3590733.

Abbreviations: IROMP, iron-regulated outer-membrane protein; OM, outer membrane.
$14 \mathrm{kDa}$ protein or the $75 \mathrm{kDa}$ IROMP were still able to transport ferri-pyochelin, indicating that both systems are operative. Heinrichs et al. (1991) suggested that the $14 \mathrm{kDa}$ system operated in exponential phase cells, whereas the $75 \mathrm{kDa}$ IROMP transporter was of greater importance in late exponential and early stationary phase.

$P$. aeruginosa also produces pyoverdins, which are structurally related to the pseudobactins from other fluorescent pseudomonads. These are highly watersoluble yellow-green compounds which all share a dihydroxyquinoline chromophore and two $N$-hydroxyornithines attached to a varying amino acid backbone of 6-10 residues (Demange et al., 1987). Evidence is now emerging that transport of different ferri-pyoverdin and ferri-pseudobactin complexes is mediated via various IROMPs, some of which have broad specificity for several complexes and some of which are specific for a single complex. It seems likely that these interactions are controlled possibly by subtle differences in the amino acid backbone of the siderophore. Amongst $P$. aeruginosa strains, Cornelis et al. (1989) noted three pyoverdin uptake groups and polyclonal antisera raised against an $80 \mathrm{kDa}$ IROMP reacted only against strains producing the same siderophore. This antiserum was subsequently shown to inhibit ferri-pyoverdin transport in this strain, confirming that the $80 \mathrm{kDa}$ IROMP was a transporter 
(Meyer et al., 1990). Poole et al. (1991) have identified a $90 \mathrm{kDa}$ IROMP ferri-pyoverdin transporter in the pyoverdin-deficient strain 6609. A mutant deficient in expression of this protein still showed low uptake of ferripyoverdin, providing evidence for a second transport system. We have obtained similar evidence in a strain made resistant to $P$. aeruginosa pyocin $\mathrm{Sa}$. This mutant lacked an $85 \mathrm{kDa}$ IROMP and was deficient in ferripyoverdin transport, but it did still show $5 \%$ residual uptake (Smith et al., 1992).

Although it is now becoming clear that $P$. aeruginosa has multiple iron transport systems, the contribution of these systems to growth in vivo is unclear. Ankenbauer et al. (1985) obtained mutants deficient in one or both siderophores and concluded that pyoverdin was more important for growth in the presence of serum or purified transferrin. However, the inability of the pyoverdindeficient $\left(\mathrm{pvd}^{-}\right)$strain to grow in serum was attributed to a failure to synthesize pyochelin, rather than to the siderophore being unable to acquire iron from transferrin.

In an attempt to understand the regulation of expression of iron-uptake systems in $P$. aeruginosa, we have been investigating iron uptake in a mutant deficient in both pyoverdin and pyochelin production. In this report, we demonstrate induction of specific IROMPs in response to growth either in the presence of pyoverdin or pyochelin and show that induction of iron uptake is siderophore-specific.

\section{Methods}

Bacterial strains and culture conditions. $P$. aeruginosa IA1, a Pvd-Pch- mutant of PAOl (ATCC 15692) obtained from Dr C. D. Cox (Ankenbauer et al., 1985) was used. PAO1 (ATCC 15692) was from the Aston Collection and 0:12, a clinical isolate from a cystic fibrosis patient, was donated by Dr Ty Pitt (Division of Hospital Infection, Public Health Laboratory Service, Colindale Avenue, London, UK). Strains were routinely maintained on nutrient agar (Oxoid) slopes at $4{ }^{\circ} \mathrm{C}$ and cultured in an iron-deficient succinate medium (Meyer \& Abdallah, 1978). Iron-replete cells were grown in succinate medium supplemented with $100 \mu \mathrm{M}-\mathrm{FeCl}_{3}$. For the induction studies, purified pyochelin or pyoverdin were added at $10 \mu \mathrm{g} \mathrm{ml}^{-1}$ and $50 \mu \mathrm{g} \mathrm{ml}^{-1}$, respectively. For outer membrane (OM) analysis and ironuptake studies, bacteria were grown for $15 \mathrm{~h}$ in an orbital shaking incubator at $37^{\circ} \mathrm{C}$ and harvested by centrifugation at $10000 \mathrm{~g}$.

Preparation of outer membranes. OMs were prepared by the Sarkosyl solubilization method of Filip et al. (1973). The washed bacterial pellet, containing approximately $2 \times 10^{11}$ cells, was suspended in $10 \mathrm{ml}$ of distilled water and broken by $10 \times 30 \mathrm{~s}$ pulses of sonication in an ice bath, with $30 \mathrm{~s}$ intervals for cooling. Unbroken cells were removed by centifugation at $10000 \mathrm{~g}$ for $10 \mathrm{~min}$. Sarkosyl ( $\mathrm{N}$-laurylsarcosinate, sodium salt; Sigma) was added to the supernate to a final concentration of $2 \%(\mathrm{w} / \mathrm{v})$. After incubation for $1 \mathrm{~h}$ at room temperature, the mixture was centrifuged at $100000 \mathrm{~g}$ for $1 \mathrm{~h}$. The OM pellet was washed twice in distilled water and stored at $-20^{\circ} \mathrm{C}$. The protein content of $\mathrm{OM}$ samples was determined by the Lowry method, with the modification that $\mathrm{OM}$ protein and bovine serum albumin standards were boiled in
$0.5 \mathrm{M}-\mathrm{NaOH}$ prior to assay. $\mathrm{OM}$ protein samples were adjusted to $1 \mathrm{mg} \mathrm{ml}^{-1}$ with distilled water.

$S D S-P A G E$. This was performed on $12 \%(\mathrm{w} / \mathrm{v})$ acrylamide gels (Lugtenberg et al., 1975) in a Mini-Protean apparatus (Bio-Rad). OM samples were mixed with an equal volume of sample buffer $(50 \mathrm{~mm}$ Tris $/ \mathrm{HCl}, \mathrm{pH} 6.8,2 \%$, w/v, SDS, $10 \%, \mathrm{v} / \mathrm{v}$, glycerol and $1 \%$, v/v, $\beta$ mercaptoethanol) and boiled at $100{ }^{\circ} \mathrm{C}$ for $10 \mathrm{~min}$ prior to electrophoresis. Gels were stained with Coomassie Brilliant blue R-250 in methanol/water/acetic acid $(50: 40: 10$, by vol.) and destained in water/methanol/acetic acid ( $83: 10: 7$, by vol.).

Immunoblotting procedure. Proteins separated by SDS-PAGE were electroblotted to nitrocellulose by the method of Towbin et al. (1979) using a Transblot apparatus (Bio-Rad). Transfer of proteins was confirmed by staining with $1 \%(\mathrm{w} / \mathrm{v})$ amido black in water/methanol/ acetic acid $\left(83: 10: 7\right.$, by vol.). The nitrocellulose was incubated at $37^{\circ} \mathrm{C}$ for $1 \mathrm{~h}$ in $5 \%(\mathrm{w} / \mathrm{v})$ skimmed milk in Tris-buffered saline (TBS; $0.9 \%$, $\mathrm{w} / \mathrm{v}, \mathrm{NaCl}$ in $10 \mathrm{mM}$-Tris/ $\mathrm{HCl}, \mathrm{pH} \mathrm{7.4)}$ to block non-specific antibody binding. The nitrocellulose paper was incubated for $4 \mathrm{~h}$ at $37^{\circ} \mathrm{C}$ with hybridoma supernatant 1:50 in TBS/milk. The blot was washed three times in TBS and incubated with 1:1000 protein A (from Staphylococcus aureus) conjugated to horseradish peroxidase (Sigma) in TBS/milk for $2 \mathrm{~h}$ at $37^{\circ} \mathrm{C}$. The antigenic components were visualized with $0.0025 \%(\mathrm{w} / \mathrm{v})$ 4-chloro-1-naphthol (Sigma) in $0.01 \%(\mathrm{v} / \mathrm{v}) \mathrm{H}_{2} \mathrm{O}_{2}$ in $10 \mathrm{~mm}-\mathrm{Tris} / \mathrm{HCl}$, at $\mathrm{pH} 7 \cdot 4$.

Purification of pyoverdin. The method was adapted from Poole et al. (1991). Cells were grown to early stationary phase in $500 \mathrm{ml}$ of succinate medium at $37^{\circ} \mathrm{C}$ and removed by centrifugation at $10000 \mathrm{~g}$ for $10 \mathrm{~min}$. The supernatant was freeze-dried, resuspended in $12 \mathrm{ml}$ of water and any undissolved matter removed by centrifugation. This solution was extracted with an equal volume of ethyl acetate and then saturated with $\mathrm{NaCl}$ prior to two extractions with $0.5 \mathrm{vol}$. phenol/chloroform (in equal parts). The aqueous phase was re-extracted and the pooled organic phases were mixed with 2 vols diethyl ether. The precipitated pyoverdin was recovered by centrifugation at $10000 \mathrm{~g}$ and washed three times with ether. Finally, the pellet was dried and dissolved in water to $20 \mathrm{mg} \mathrm{ml}^{-1}$.

${ }^{55} \mathrm{Fe}$-Pyoverdin uptake. The general procedure of Poole et al. (1991) was used. Cells were grown in succinate medium to late exponential phase and harvested by centrifugation at $4{ }^{\circ} \mathrm{C}$. The cells were washed twice with succinate medium, resuspended to an $\mathrm{OD}_{470}$ of 1.0 , corresponding to $10^{9}$ cells, and equilibrated at $37^{\circ} \mathrm{C}$ for $15 \mathrm{~min}$ prior to transport assays. During equilibration, no observable increase in $\mathrm{OD}_{470}$ was observed. Pyoverdin $(80 \mu \mathrm{g})$ and $115 \mathrm{nM}^{-55} \mathrm{FeCl}_{3}$ [32.18 $\mathrm{mCi} \mathrm{mg}^{-1}\left(1.91 \mathrm{GBq} \mathrm{mg}^{-1}\right)$ Amersham] were added to a $1 \mathrm{ml}$ volume of cell suspension at $37^{\circ} \mathrm{C}$. Ferri-pyoverdin uptake was assayed by withdrawing $200 \mu \mathrm{l}$ samples and filtering through $0.2 \mu \mathrm{m}$ cellulose acetate membranes (Whatman). The membranes were washed twice with $10 \mathrm{ml}$ volumes of saline and allowed to air-dry. The activity retained on the membranes was determined by scintillation counting on the ${ }^{3} \mathrm{H}$ channel of a Packard TR 1600 betacounter using Optiphase Hisafe II scintillant (LKB-Bromma).

Purification of pyochelin. The procedure of Meyer et al. (1989) was adapted as follows. Cells were grown to early stationary phase in Casamino acids medium (Ankenbauer et al., 1985), removed by centrifugation and the supernatant acidified to $\mathrm{pH} 3.0$ with glacial acetic acid. After extraction with 0.5 vol. ethyl acetate, the organic phase was evaporated to dryness under reduced pressure. The crudely purified pyochelin was dissolved in $1 \mathrm{ml}$ of methanol. This preparation was purified further by chromatography through a $45 \times 1.5 \mathrm{~cm}$ column of Sephadex LH-20 (Pharmacia) in methanol. Fractions were spotted onto Whatman $3 \mathrm{MM}$ paper and sprayed with $0 \cdot 1 \mathrm{M}-\mathrm{FeCl}_{3}$ in $0 \cdot 1 \mathrm{M}-\mathrm{HCl}$ in order to detect iron chelating activity. Positive fractions were stored dry at $4{ }^{\circ} \mathrm{C}$. 


\begin{abstract}
${ }^{55} \mathrm{Fe}$-Pyochelin uptake. The cells were harvested and washed as described above for the ${ }^{55} \mathrm{Fe}$-pyoverdin uptake studies. Pyochelin (10 mM in methanol) and ${ }^{55} \mathrm{FeCl}_{3}$ were added to final concentrations of $20 \mu \mathrm{g} \mathrm{ml}^{-1}$ and $115 \mathrm{nM}$, respectively, in succinate medium $15 \mathrm{~min}$ prior to the start of an uptake experiment. Uptake was initiated by addition of cells at $37^{\circ} \mathrm{C}$ to a final $\mathrm{OD}_{470}$ of 1.0 . Cell filtration and scintillation counting were as described above, except that cells were washed with $0.5 \mathrm{M}-\mathrm{HCl}$.
\end{abstract}

\section{Results}

\section{Analysis of IROMPS}

Preliminary experiments in which crude supernatants from cultures of 17 other cystic-fibrosis-derived clinical isolates of $P$. aeruginos $a$ were added to IA1 indicated that growth could be either increased or inhibited depending on the strain, suggesting that IA 1 could be cross-fed from some siderophores, but not from others. In order to assess whether these effects were reflected in changes in OM protein profiles, siderophores were purified and added to cultures of IAl in succinate medium. The IROMP profiles are shown in Fig. 1. In succinate medium alone, IA1 strongly expressed three IROMPs of 81,83 and $96 \mathrm{kDa}$ (lane 1), whereas addition of pyoverdin from the parental strain PAO1 (lane 2) caused induction of an $85 \mathrm{kDa}$ IROMP and partially repressed the 96,83 and $81 \mathrm{kDa}$ IROMPs. Addition of chromatographically distinct pyoverdin from strain $0: 12$ (lane 3) induced the $85 \mathrm{kDa}$ IROMP, and partially repressed the $81 \mathrm{kDa}$ IROMP. Addition of purified pyochelin increased expression of a $75 \mathrm{kDa}$ IROMP only (lane 4). Growth in iron-replete succinate medium (lane 5) repressed all high molecular mass IROMPs.
Fig. 2 shows a Western blot of the IROMPs reacted with $\mathrm{mAb} \mathrm{C108}$, which was raised against an $85 \mathrm{kDa}$ IROMP from strain AK1282, an LPS-defective derivative of PAO1 (Smith et al., 1991). No reaction was seen against IROMPs prepared from iron-replete cells (lane 5). Weak activity was seen against IROMPs of IAl alone (lane 1) or supplemented with pyochelin (lane 4), whereas a strong reaction with some cross-reactivity with lower molecular mass proteins was seen against IROMPs from cells grown in the presence of added pyoverdins (lanes 2 and 3).

\section{Ferri-pyoverdin uptake after growth with pyochelin or pyoverdin}

Since cross-feeding with purified pyoverdins caused changes in IROMP profiles, labelled-iron transport studies were performed to determine whether ferrisiderophore uptake was affected. Fig. 3 shows iron uptake from ferri-pyoverdin after growth in succinate medium with and without addition of pyoverdin or pyochelin. Growth in the presence of pyoverdin caused a $2 \cdot 8$-fold increase in the initial rate of iron uptake from ferri-pyoverdin. The rate increased from $1.27 \mathrm{pmol} \mathrm{Fe}$ $\mathrm{min}^{-1}$ per $10^{9}$ cells without induction to $3.57 \mathrm{pmol} \mathrm{Fe}$ $\min ^{-1}$ per $10^{9}$ cells with growth in the presence of pyoverdin. Growth in medium supplemented with pyochelin had no effect on ferri-pyoverdin uptake. Preincubation of cells with $\mathrm{mAb} \mathrm{C} 108$ hybridoma supernatant diluted 1:50 in succinate medium during the $15 \mathrm{~min}$ equilibration period at $37^{\circ} \mathrm{C}$ did not affect uptake (data not shown).

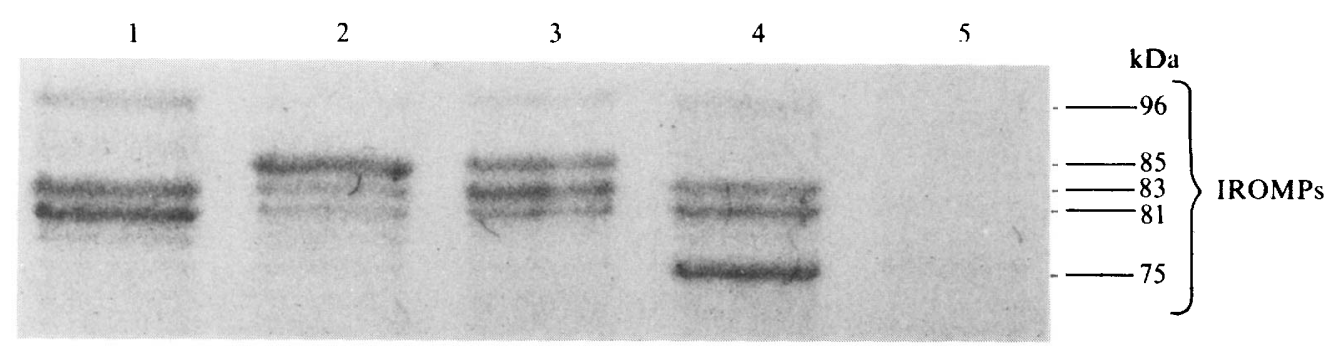

Fig. 1. SDS-PAGE of OMs prepared from $P$. aeruginosa IA1 grown in iron-deficient succinate medium (lane 1), supplemented with

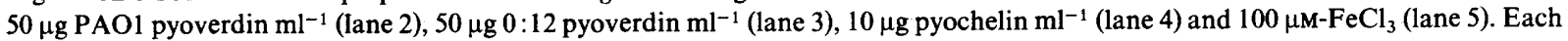
lane contained $5 \mu \mathrm{g}$ protein.

1

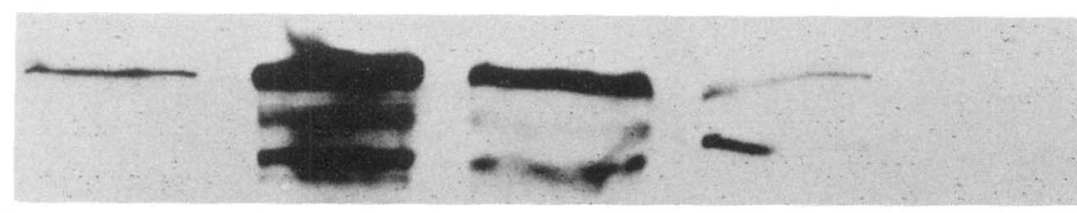

Fig. 2. Immunoblot probed with mAb C108. Lanes are as described in Fig. 1. 


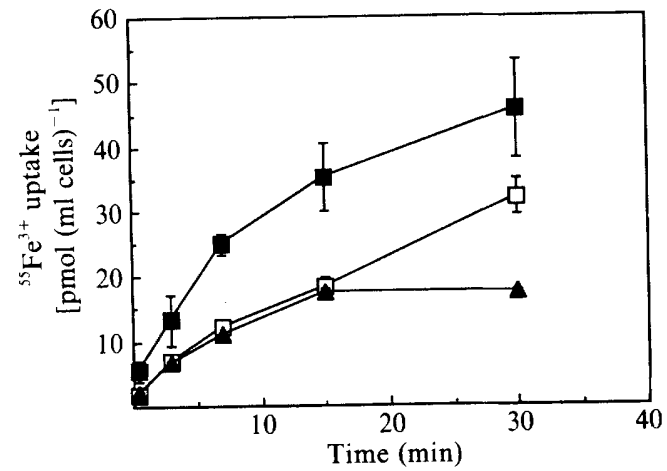

Fig. 3

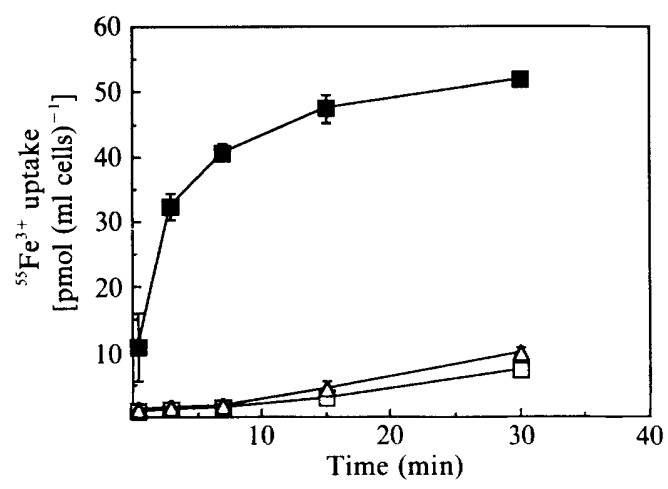

Fig. 4

Fig. 3. PAO1-pyoverdin-mediated iron $\left({ }^{55} \mathrm{Fe}^{3+}\right)$ uptake by $P$. aeruginosa IAl grown in iron-deficient succinate medium ( $\square$ ), supplemented with $50 \mu \mathrm{g}$ PAOl pyoverdin $\mathrm{ml}^{-1}(\mathbf{G})$ or supplemented with $10 \mu \mathrm{g}$ pyochelin $\mathrm{ml}^{-1}(\boldsymbol{\Lambda})$. The uptake mixture contained pyoverdin $\left(80 \mu \mathrm{g} \mathrm{ml}^{-1}\right),{ }^{55} \mathrm{FeCl}_{3}(115 \mathrm{nM})$ and $1 \mathrm{ml}$ of cells at an $\mathrm{OD}_{470}$ of $1 \cdot 0$. Experiments were performed in triplicate and the results expressed as the mean $\pm \mathrm{SD}$.

Fig. 4. Pyochelin-mediated iron $\left({ }^{55} \mathrm{Fe}^{3+}\right)$ uptake by $P$. aeruginosa IAl grown in iron-deficient succinate medium ( $\square$ ), supplemented with $50 \mu \mathrm{g}$ PAOl pyoverdin $\mathrm{ml}^{-1}(\triangle)$ or supplemented with $10 \mu \mathrm{g}$ pyochelin $\mathrm{ml}^{-1}(\mathbb{\square})$. The uptake mixture contained pyochelin $\left(20 \mu \mathrm{g} \mathrm{ml}^{-1}\right),{ }^{55} \mathrm{FeCl}_{3}(115 \mathrm{nM})$ and $1 \mathrm{ml}$ of cells at an $\mathrm{OD}_{470}$ of 1.0 . Experiments were performed in triplicate and the results expressed as the mean $\pm \mathrm{SD}$.

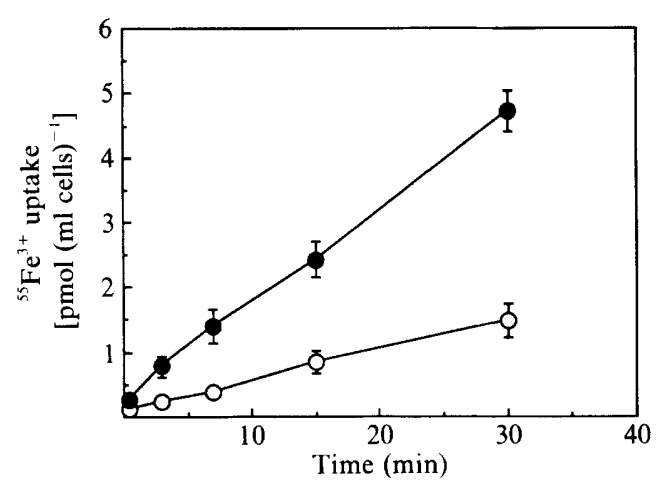

Fig. 5

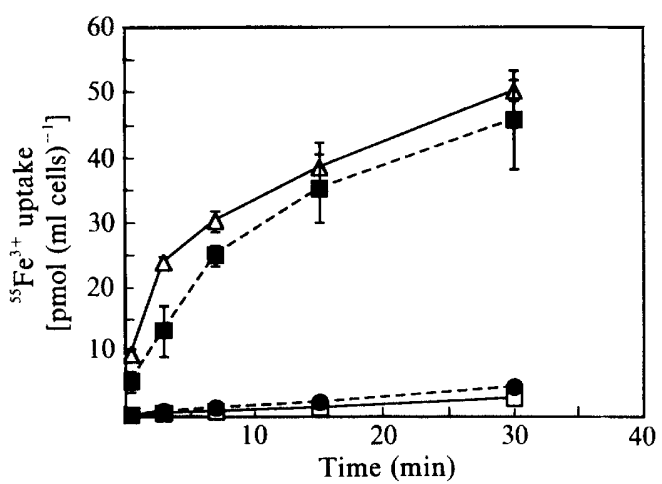

Fig. 6

Fig. 5. 0:12-pyoverdin-mediated iron $\left({ }^{55} \mathrm{Fe}^{3+}\right)$ uptake by $P$. aeruginosa IAI grown in iron-deficient succinate medium alone $(\mathrm{O})$ or supplemented with $50 \mu \mathrm{g} \mathrm{0:12} \mathrm{pyoverdin} \mathrm{ml}^{-1}(\bigcirc)$. The uptake mixture contained pyoverdin $\left(80 \mu \mathrm{g} \mathrm{ml}^{-1}\right),{ }^{55} \mathrm{FeCl}_{3}(115 \mathrm{nM})$ and $1 \mathrm{ml}$ of cells at an $\mathrm{OD}_{470}$ of 1.0 . Experiments were performed in triplicate and the results expressed as the mean \pm SD.

Fig. 6. Pyoverdin cross-induction and uptake studies in P. aeruginosa IA1. Pyoverdin supplementation of iron-deficient succinate growth medium and iron $\left({ }^{55} \mathrm{Fe}^{3+}\right)$ uptake studies were performed as follows: induction with $0: 12$ and uptake via PAO1 pyoverdins $(\triangle)$; induction with PAO1 and uptake via PAOl pyoverdin ( $\boldsymbol{\square})$; induction with $0: 12$ and uptake via $0: 12$ pyoverdin (O); induction with PAO1 and uptake via $0: 12$ pyoverdins $(\square)$. The uptake mixture contained pyoverdin $\left(80 \mu \mathrm{g} \mathrm{ml}^{-1}\right),{ }^{55} \mathrm{FeCl}_{3}(115 \mathrm{nM})$ and $1 \mathrm{ml}$ of cells at an $\mathrm{OD}_{470}$ of 1.0 . The dashed lines are duplicated from Figs. 3 and 5 for comparison. Experiments were performed in triplicate and the results expressed as the mean \pm SD.

\section{Ferri-pyochelin uptake after growth with pyochelin or pyoverdin}

Fig. 4 shows the results of ferri-pyochelin uptake studies. Similar to the observations made for ferri-pyoverdin uptake, ferri-pyochelin uptake was increased only after growth with pyochelin. The increase in initial rate of

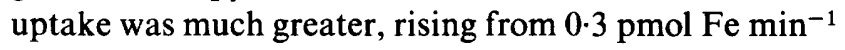

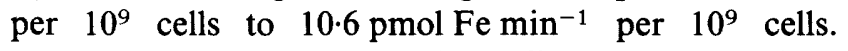
Pyoverdin pre-treatment had no effect, the rate of iron uptake being $0 \cdot 3$ pmol Fe $\min ^{-1}$ per $10^{9}$ cells.

\section{Pre-treatment with chromatographically distinct pyoverdins}

Pyoverdins from eight strains in the laboratory collection were purified and analysed by thin-layer chromatography in methanol/water $(70: 30, \mathrm{v} / \mathrm{v})$ as described by Bitter et al. (1991). The $R_{F}$ values for PAO1 and $0: 12$ pyoverdins were 0.38 and 0.22 , respectively. Ferripyoverdin uptake profiles for $\boldsymbol{P}$. aeruginosa IA1 using pyoverdin from strain $0: 12$, with and without growth in the presence of this siderophore, are shown in Fig. 5. 
Increased uptake was noted with cells grown in the presence of siderophore, but the rate and extent of uptake, even after pre-treatment, were significantly less than that seen with PAO1 pyoverdin (Fig. 3). The pretreatment experiments were repeated using either PAO1 or $0: 12$ pyoverdin and uptake experiments performed with $0: 12$ and PAO1 pyoverdins, respectively. Fig. 6 shows the uptake profiles. The increased uptake was the same, irrespective of which pyoverdin had been added to the growth medium.

\section{Discussion}

In this work we have shown that $P$. aeruginosa can respond specifically to the presence of siderophore in the growth medium and not simply to the iron-deficiency imposed by that siderophore. This is based on the finding that growth in the presence of pyoverdin induces ferripyoverdin uptake only and growth in the presence of pyochelin increases ferri-pyochelin uptake only. This may go some way to explaining why $P$. aeruginosa retains two siderophore-mediated iron-uptake systems, despite the observation that the binding constant of iron for pyoverdin $\left(10^{32}\right)$ (Wendenbaum et al., 1983) is significantly greater than that of pyochelin $\left(10^{5}\right.$ at acid $\left.\mathrm{pH}\right)$, which in addition requires two molecules to bind one molecule of iron (Cox \& Graham, 1979). We speculate that $P$. aeruginosa produces low amounts of each siderophore in iron-deficient growth environments and monitors which siderophore is most effective at complexing iron before becoming committed to, and inducing, iron uptake via that route. Although the observations reported in this work were made with the siderophoredeficient strain IA1, we also noted inducible uptake with the siderophore-competent parent strain PAOl (data not shown).

Our finding that the increased uptake of ferripyochelin after growth in pyochelin-supplemented medium correlated with increased expression of a $75 \mathrm{kDa}$ IROMP supports the finding of Heinrichs et al. (1991). These workers noted increased expression of a $75 \mathrm{kDa}$ IROMP in PAO 6609, a pyoverdin-biosynthesisdeficient mutant of PAO1. They also showed that strain K372, a pirazmonam-resistant mutant of PAO 6609 which lacks this $75 \mathrm{kDa}$ IROMP, failed to transport ferri-pyochelin. We did not observe any changes in expression of the $14 \mathrm{kDa}$ ferri-pyochelin binding protein (Sokol \& Woods, 1983), although our studies were carried out using early stationary phase cells when this uptake system is thought to be less important (Heinrichs et al., 1991).

Several groups have reported IROMPs in the range $80-90 \mathrm{kDa}$ that are associated with ferri-pyoverdin uptake in different strains (Cornelis et al., 1989; Meyer et al., 1990; Poole et al., 1991). In this work, ferri-pyoverdin uptake correlated with expression of an $85 \mathrm{kDa}$ IROMP, although we were unable to inhibit uptake with the anti$85 \mathrm{kDa}$ IROMP mAb. However, in previous studies we have shown only poor interaction with whole cells, possibly as a result of being shielded by lipopolysaccharide or because the mAb was raised against an epitope which is not surface-exposed (Smith et al., 1991). It was interesting to note that uptake by IA 1 of ferri-pyoverdins from other strains occurred at different rates, suggesting that either the receptor for its own pyoverdin had a broader substrate specificity, with not all ferri-pyoverdin complexes being taken up at the same rate, or that a second ferri-pyoverdin receptor exists. The induction studies with different pyoverdins, from PAO1 and strain $0: 12$, suggest that the same receptor is induced (Fig. 1, lanes 1,2 and 3), and this was supported further by the increased reactivity with the anti-85 $\mathrm{kDa}$ IROMP $\mathrm{mAb}$ (Fig. 2, lanes 1, 2 and 3). Pre-treatment with either pyoverdin resulted in the same rate of uptake; pretreatment with $0: 12$ pyoverdin resulted in the same induction of PAOl ferri-pyoverdin uptake as pretreatment with PAO1 pyoverdin itself. The reverse was also true, with PAO1 pyoverdin pre-treatment resulting in the same induction of $0: 12$ ferri-pyoverdin uptake as with $0: 12$ pyoverdin itself. Competition studies with ${ }^{55} \mathrm{Fe}-0: 12$ pyoverdin and non-radioactive Fe-PAO1 complex proved inconclusive (data not shown). It was expected that the rate of uptake of ${ }^{55} \mathrm{Fe}-0: 12$ complex would be reduced in the presence of $\mathrm{Fe}-\mathrm{PAO} 1$ pyoverdin complex due to competition at the receptor. However, the opposite was observed. This was attributed to transport of ${ }^{55} \mathrm{Fe}$ as the ${ }^{55} \mathrm{Fe}-\mathrm{PAO} 1$ complex, which resulted from equilibration between the two pyoverdins. These data suggest therefore that with the pyoverdins and strains used in this study, the uptake and induction phenomena require just one receptor. However, it cannot be ruled out that there is a second uptake system, and the functions of other high molecular mass IROMPs, notably the 81 and $83 \mathrm{kDa}$ proteins, remain to be determined. Poole et al. (1991) have noted residual uptake of ferri-pyoverdin complex in a mutant deficient in a $90 \mathrm{kDa}$ IROMP and suggested that this is due to a second transport system. Interestingly, these workers were not able to demonstrate an induction phenomenon in this strain. Similarly, we were unable to demonstrate induction in a strain rendered unable to transport ferripyoverdin through resistance to $P$. aeruginosa pyocin $\mathrm{Sa}$ (Smith et al., 1992). Both these strains lacked a major IROMP associated with ferri-pyoverdin uptake, which suggests that the IROMP must be present for the induction phenomenon reported in this work to be seen.

Poole et al. (1990) have noted an induction pheno- 
menon in $P$. aeruginosa, but with the enterobacterial siderophore enterobactin. Growth inhibition of a pyoverdin-deficient strain imposed by the non-metabolizable chelator ethylene diamine-di(o-hydroxyphenol acetic acid) could be reversed by addition of enterobactin. A novel $80 \mathrm{kDa}$ IROMP was detected and enterobactingrown cells transported ferri-enterobactin in an energydependent manner at low iron concentrations. Leong et al. (1991) have noted an induction response to exogenous siderophore in the related fluorescent pseudomonad $P$. putida. In their study, the gene coding for a second pseudobactin uptake system (pupB), located on plasmid pMK15, was identified by its ability to render transformed strains no longer susceptible to starvation by pseudobactin BN7, the native siderophore of strain BN7. However, an additional OM protein was expressed in strain A124/pMK 15 only after growth in the presence of pseudobactin BN7. Moreover, these workers noted new OM proteins when strains were grown in the presence of pseudobactin $\mathrm{B} 10$ and pyoverdin, but not deferriferrichrome A. Our studies extend these observations to $P$. aeruginosa and to siderophores which are not structurally related, i.e. pyoverdin and pyochelin. At the present stage, it is unclear how the signal of siderophore present in the growth medium is detected by the cell, but evidence is now emerging for positive and negative iron regulators in $P$. aeruginosa. Prince et al. (1991) have identified a Fur homologue in $P$. aeruginosa and have also noted that the $E$. coli fur gene will regulate exotoxin A expression. Several workers have identified positive regulators of iron-regulated gene expression, notably the regA gene in exotoxin A expression (Wick et al., 1990) and LasR regulation of elastase expression (Gambello \& Iglewski, 1991) in $P$. aeruginosa, and a transcriptional activator associated with a gene from the siderophore biosynthesis/uptake cluster of a fluorescent plant pseudomonad (O'Sullivan \& O'Gara, 1991).

In summary we have shown that $P$. aeruginosa can respond specifically to the presence either of pyoverdin or pyochelin in the growth medium. We are currently cloning siderophore biosynthesis/uptake genes to determine how this response is regulated.

The authors wish to thank Adrian Lowe for technical assistance. This work was supported by grants from the Nuffield Foundation (A.W.S.), Royal Society (A.W.S.) and a Science and Engineering Research Council postgraduate studentship (K.G.).

\section{References}

Ankenbauer, R., SRiyosachati, S. \& Cox, C. D. (1985). Effects of siderophores on the growth of Pseudomonas aeruginosa in human serum and transferrin. Infection and Immunity 46, 132-140.

Bitter, W., Marugg, J. D., De Weger, L. A., Tommassen, J. \& WeISBEeK, M. P. J. (1991). The ferric-pseudobactin receptor PupA of Pseudomonas putida WCS358: homology to TonB-dependent Escher- ichia coli receptors and specificity of the protein. Molecular Microbiology 5, 647-655.

Cornelis, P., Hohnadel, D. \& Meyer, J. M. (1989). Evidence for different pyoverdin-mediated iron uptake systems among Pseudomonas aeruginosa strains. Infection and Immunity 57, 3491-3497.

Cox, C. D. \& Graham, R. (1979). Isolation of an iron-binding compound from Pseudomonas aeruginosa. Journal of Bacteriology 93, 144-148.

Cox, C. D., Rinehart, K. L., Moore, M. L. \& CoOK, J. C. (1981). Pyochelin: novel structure of an iron-chelating growth promoter from Pseudomonas aeruginosa. Proceedings of the National Academy of Sciences of the United States of America 78, 302-308.

Demange, P., Wendenbaum, S., Bateman, A., Dell, A. \& Abdallah, M. A. (1987). Bacterial siderophores: structure and physicochemical properties of pyoverdins and related compounds. In Iron Transport in Microbes, Plants and Animals, pp. 189-205. Edited by G. Winkelmann. Weinheim: VCH.

Filip, C., Fletcher, G., WulfF, J. L. \& Earhart, C. F. (1973). Solubilization of the outer membrane of Escherichia coli by the ionic detergent sodium-lauryl sarcosinate. Journal of Bacteriology 115, 717-722.

GAMBELLO, M. J. \& IGLEWSKI, B. H. (1991). Cloning and characterization of the Pseudomonas aeruginosa lasR gene, a transcriptional activator of elastase expression. Journal of Bacteriology 173, 30003009.

GRIFFITHS, E. (1991). Iron and bacterial virulence - a brief overview. Biology of Metals 4, 7-13.

HeinRIChs, D. A., Young, L. \& Poole, K. (1991). Pyochelin-mediated iron transport in Pseudomonas aeruginosa: involvement of a highmolecular-mass outer membrane protein. Infection and Immunity 59 $3680-3684$.

Leong, J., Bitter, W., Koster, M., Venturi, V. \& Weisbeek, P. J. (1991). Molecular analysis of iron transport in plant growthpromoting Pseudomonas putida WCS358. Biology of Metals 4, 36-40.

Lugtenderg, B., Meijers, J., Peters, R., Van Der Hoeck, P.\& Van ALPHEN, L. (1975). Electrophoretic resolution of the "major outer membrane protein' of Escherichia coli K-12. FEBS Letters 58, 254258.

MeYer, J. M. \& ABDallah, M. A. (1978). The fluorescent pigment of Pseudomonas fluorescens: biosynthesis, purification and physicochemical properties. Journal of General Microbiology 107, 319328.

MeYer, J. M., Hohnadel, D. \& Halle, F. (1989). Cepabactin from Pseudomonas cepacia, a new type of siderophore. Journal of General Microbiology 135, 1479-1487.

Meyer, J. M., Hohnadel, D., Khan, A. \& Cornelis, P. (1990). Pyoverdin-facilitated iron uptake in Pseudomonas aeruginosa: immunological characterization of the ferripyoverdin receptor. $\mathrm{Molecu}$ lar Microbiology 4, 1401-1405.

O'Sullivan, D. J. \& O'GaRA, F. (1991). Regulation of iron assimilation: nucleotide sequence of an iron-regulated promoter from a fluorescent pseudomonad. Molecular and General Genetics 228, $1-8$.

Poole, K., Young, L. \& Neshat, S. (1990). Enterobactin-mediated iron transport in Pseudomonas aeruginosa. Journal of Bacteriology 172, 6991-6996.

Poole, K., Neshat, S. \& Heinrichs, D. (1991). Pyoverdin-mediated iron transport in Pseudomonas aeruginosa. FEMS Microbiology Letters 78, 1-6.

Prince, R. W., Storey, D. G., Vasil, A. I. \& Vasil, M. L. (1991). Regulation of $\operatorname{tox} A$ and $\operatorname{reg} A$ by the Escherichia coli fur gene and identification of a Fur homologue in Pseudomonas aeruginosa PA103 and PAO1. Molecular Microbiology 5, 2823-2831.

Smith, A. W., Hirst, P. H., Hughes, K., Gensberg, K. \& Govan, J. R. W. (1992). The pyocin Sa receptor of Pseudomonas aeruginosa is associated with ferripyoverdin uptake. Journal of Bacteriology 174, $4847-4849$.

Smith, A. W., Wilton, J., Clark, S. A., Alpar, O., Melling, J. \& BrowN, M. R. W. (1991). Production and characterization of monoclonal antibodies to outer membrane proteins of Pseudomonas aeruginosa grown in iron-depleted media. Journal of General Microbiology 137, 227-236. 
Sokol, P. A. (1987). Tn5 insertion mutants of Pseudomonas aeruginosa deficient in surface expression of ferripyochelin-binding protein. Journal of Bacteriology 169, 3365-3368.

Sokol, P. A. \& Woods, D. E. (1983). Demonstration of an ironsiderophore-binding protein in the outer membrane of Pseudomonas aeruginosa. Infection and Immunity 40, 665-669.

Towbin, H., Staehelin, T. \& Gordon, J. (1979). Electrophoretic transfer of proteins from polyacrylamide gels to nitrocellulose sheets: procedure and some applications. Proceedings of the National
Academy of Sciences of the United States of America 76, 43504354.

Wendenbaum, S., Demange, P., Dell, A., Meyer, J. M. \& AbDallaH, M. A. (1983). The structure of pyoverdine $P a$, the siderophore of Pseudomonas aeruginosa. Tetrahedron Letters 24, 4877-4880.

Wick, M. J., Frank, D. W., Storey, D. G. \& IGlewski, B. H. (1990). Structure, function and regulation of Pseudomonas aeruginosa exotoxin A. Annual Review of Microbiology 44, 335-363. 